TRANSACTIONS OF THE

AMERICAN MATHEMATICAL SOCIETY

Volume 363, Number 12, December 2011, Pages 6453-6463

S 0002-9947(2011)05308-0

Article electronically published on July 25, 2011

\title{
RATIONAL MAPS WITH REAL MULTIPLIERS
}

\author{
ALEXANDRE EREMENKO AND SEBASTIAN VAN STRIEN
}

\begin{abstract}
Let $f$ be a rational function such that the multipliers of all repelling periodic points are real. We prove that the Julia set of such a function belongs to a circle. Combining this with a result of Fatou we conclude that whenever $J(f)$ belongs to a smooth curve, it also belongs to a circle. Then we discuss rational functions whose Julia sets belong to a circle.
\end{abstract}

A simple argument of Fatou [4, Section 46] shows that if the Julia set of a rational function is a smooth curve, then all periodic orbits on the Julia set have real multipliers; see also [8, Cor. 8.11]. This argument gives the same conclusion if one only assumes that the Julia set is contained in a smooth curve. By a smooth curve we mean a curve that has a tangent at every point. All rational functions in this paper are supposed to have degree at least 2 .

We prove the converse statement:

Theorem 1. Let $f: \overline{\mathbb{C}} \rightarrow \overline{\mathbb{C}}$ be a rational map such that the multiplier of each repelling periodic orbit is real. Then either the Julia set $J(f)$ is contained in a circle or $f$ is a Lattès map.

Corollary 1. If the Julia set of a rational function is contained in a smooth curve, then it is contained in a circle.

In fact, Theorem 1 holds if all repelling periodic points on some relatively open subset of $J(f)$ have real multipliers. It follows that even if a relatively open set of the Julia set is contained in a smooth curve, then the Julia set is contained in a circle.

This corollary generalizes the result of Fatou [4, Section 43] that whenever the Julia set of a rational function is a smooth curve, this curve has to be a circle or an arc of a circle. For another proof of the corollary, independent of Theorem 1, see [1]. We give a more precise description of the maps which can occur:

Theorem 2. Let $f: \overline{\mathbb{C}} \rightarrow \overline{\mathbb{C}}$ be a rational map whose Julia set $J(f)$ is contained in a circle $C$. Then there are the following possibilities:

(i) $C$ is completely invariant, in which case $f^{2}$ is a Blaschke product (that is, both components of the complement of $C$ are invariant under $\left.f^{2}\right)$. The Julia set is either $C$ or a Cantor subset of $C$.

If (i) does not hold, then there is a critical point on $C$ and a fixed point $x_{0} \in C$ whose multiplier satisfies $\lambda \in[-1,1]$. Let $I$ be the smallest closed arc on $C$ which

Received by the editors November 13, 2008 and, in revised form, December 15, 2009.

2010 Mathematics Subject Classification. Primary 37F10, 30D05.

The first author was supported by NSF grant DMS-0555279.

The second author was supported by a Royal Society Leverhulme Trust Senior Research Fellowship.

(C)2011 American Mathematical Society Reverts to public domain 28 years from publication 
contains $J(f)$ and whose interior does not contain $x_{0}$. Then one of the following holds:

(ii) $I$ is a proper arc which is completely invariant, and $J(f)=I$.

(iii) $f(I)$ strictly contains I. The Julia set is a Cantor subset of I in this case.

In cases (ii) and (iii), for each critical point $x$ of $f$ in I there exists $N \geq 1$ such that $f^{N}(x) \notin$ interior $(I)$ (where in case (ii) $N=1$ ). All critical points on $J(f)$ are preperiodic.

Remarks. If $f$ is a Blaschke product preserving a circle $C$, then $f: C \rightarrow C$ is a covering, so cases (i), (ii) and (iii) are disjoint. Chebyshev polynomials belong to case (ii), and every polynomial satisfying (ii) is conjugate to $\pm T$, where $T$ is a Chebyshev polynomial. If $f$ in case (iii) is a polynomial, one can always take $N=1$, but there are rational functions satisfying (iii) for which $N>1$; see Example 3 at the end of the paper.

There are functions $f$ satisfying (ii) which are not conjugate to polynomials. A parametric description of functions satisfying (ii) can be obtained using [3, Section 25] 1

Each $f$ satisfying (ii) is conjugate to $B^{2}(\sqrt{z})$, where $B$ is an odd rational function which leaves both upper and lower half-planes invariant, and whose Julia set equals $\overline{\mathbb{R}}$. In the opposite direction, if $B$ is an odd rational function which leaves both upper and lower half-planes invariant, and whose Julia set equals $\overline{\mathbb{R}}$, then $B^{2}(\sqrt{z})$ is a rational function whose Julia set is the ray $[0, \infty]$, and this function satisfies (ii).

In cases (ii) and (iii) the interval $I$ is equal to $C \backslash B_{0}$, where $B_{0}$ denotes the immediate basin of $x_{0}$ for the restriction of $f$ on $C$. In case (iii) there exist finitely many closed arcs on $C$ such that the full preimage of their union is contained in this union. To prove this claim, take (within $C$ ) the preimages up to order $N$ of $B_{0}$, where $N$ is as in Theorem 2, and therefore the union $K$ of the closures of these intervals contains all critical values in $I$. Hence $I \backslash K$ has the following properties: the closure of $I \backslash K$ contains the Julia set. As $K$ is forward invariant, $\overline{\mathbb{C}} \backslash K$ is backward invariant. As every point of $J(f)$ (and therefore every point of $I \backslash K$ ) has all preimages in the closure of $I \backslash K$, and there are no critical values in $I \backslash K$, we conclude that the closure of $I \backslash K$ is backward invariant.

Theorem 1 is proved in Section 1. In Section 2, we prove Theorem 2 and discuss rational functions satisfying (iii) of Theorem 2 .

The authors would like to thank the referee for some helpful comments on an earlier version of the paper.

\section{Proof of Theorem 1}

There are only finitely many repelling cycles which belong to the forward orbits of critical points. So there exists a repelling periodic point $p$ of $f$ of period $N$ which does not lie on the forward orbit of a critical point. Replacing $f$ by $f^{N}$ we may assume that $N=1$.

Let $\Psi: \mathbb{C} \rightarrow \overline{\mathbb{C}}$ be a holomorphic map which globally linearizes $f$ at $p$, i.e.,

$$
\Psi \lambda=f \Psi, \quad \Psi(0)=p, \quad D \Psi(0) \neq 0 .
$$

\footnotetext{
${ }^{1}$ We use this opportunity to note that the statement of these results of Fatou in the survey [2] is wrong. See Fatou's original paper for the correct statements.
} 
Here $\lambda$ is the multiplier of $p$, so $\lambda:=D f(p)$ is real. Such a map $\Psi$, which is also called a Poincaré function [11, always exists. It is uniquely defined by the value $D \Psi(0) \in \mathbb{C} \backslash\{0\}$ which can be prescribed arbitrarily.

Lemma 1. If $z \in \Psi^{-1}(p)$ and $p$ is not an iterate of a critical point, then $D \Psi(z) \neq 0$.

Proof. Since $\Psi(z)=f^{n} \Psi \lambda^{-n} z$ and $\Psi$ is univalent in a neighborhood of 0 , the result follows.

We will use the following result of Ritt [10] several times:

Lemma 2. The Poincaré function is periodic if and only if $f$ is a Lattès map, or conjugate to $\pm T_{n}$, where $T_{n}$ is a Chebyshev polynomial, or conjugate to $z^{ \pm d}$.

Rational functions with periodic Poincaré functions described in Lemma 2 will be called exceptional.

1.1. The linearizing map restricted to certain lines. A simple curve $\gamma$ : $(0,1) \rightarrow \overline{\mathbb{C}}$ passing through a repelling periodic point $p$ of period $N$ is called an unstable manifold for $p$ if there exists a subarc $\gamma_{*}$ of $\gamma$ containing $p$ so that $f^{N}$ maps $\gamma_{*}$ diffeomorphically onto $\gamma$. Similarly, we say that $\gamma$ is an invariant curve for $p_{-m} \in f^{-m}(p)$ if $\gamma$ is contained in an unstable manifold for $p, p_{-m} \in \gamma$ and $f^{m}\left(V_{m} \cap \gamma\right) \subset \gamma$ for some neighborhood $V_{m}$ of $p_{-m}$.

Choose $Q \in \Psi^{-1}(p) \backslash\{0\}$. By Lemma 1 $D \Psi(Q) \neq 0$, so there exist small topological discs $\mathcal{O}_{0} \ni 0$ and $\mathcal{O}_{1} \ni Q$ so that $\Psi \mid \mathcal{O}_{0}$ and $\Psi \mid \mathcal{O}_{1}$ are univalent and $\Psi\left(\mathcal{O}_{0}\right)=\Psi\left(\mathcal{O}_{1}\right)$. Hence there exists a biholomorphic map $\mathcal{T}: \mathcal{O}_{0} \rightarrow \mathcal{O}_{1}$ for which

$$
\mathcal{T}(0)=Q \text { and } \Psi \circ \mathcal{T}=\Psi \text { restricted to } \mathcal{O}_{0} .
$$

For convenience we may choose $\mathcal{O}_{i}$ so that $\lambda^{-1} \mathcal{O}_{0} \subset \mathcal{O}_{0}$; for example, we can take a round disc for $\mathcal{O}_{0}$.

First we show that the linearizing map $\Psi$ is special on certain lines. To prove this, we shall use the following

Lemma 3. For each $Q \in \Psi^{-1}(p) \backslash\{0\}$, there exists a sequence $z_{n} \rightarrow 0$ so that $\lambda^{n} z_{n} \rightarrow Q$ and $\Psi\left(z_{n}\right)$ is a repelling periodic point of period $n$. There exists a neighborhood $V_{n} \subset \mathcal{O}_{0}$ of $z_{n}$ so that $f^{n}: \Psi\left(V_{n}\right) \rightarrow \Psi\left(\mathcal{O}_{0}\right)$ is biholomorphic.

Proof. Take $n$ so large that the closure of $\lambda^{-n} \mathcal{O}_{1}$ is contained in $\mathcal{O}_{0}$. Notice that

$$
f^{n} \mid \Psi\left(\lambda^{-n} \mathcal{O}_{1}\right)=\left(\Psi \mid \mathcal{O}_{1}\right) \circ \lambda^{n} \circ\left(\Psi \mid \lambda^{-n} \mathcal{O}_{1}\right)^{-1},
$$

and therefore $f^{n} \mid \Psi\left(\lambda^{-n} \mathcal{O}_{1}\right)$ is univalent on $V_{n}:=\lambda^{-n} \mathcal{O}_{1}$. Moreover,

$$
f^{n}\left(\Psi\left(\lambda^{-n} \mathcal{O}_{1}\right)\right)=\Psi\left(\mathcal{O}_{1}\right)=\Psi\left(\mathcal{O}_{0}\right),
$$

and thus there exists $z_{n} \in V_{n}$ so that $\Psi\left(z_{n}\right)$ is a fixed point of $f^{n}$. As $\mathcal{O}_{0}$ and $\mathcal{O}_{1}$ can be chosen arbitrarily small, we obtain a sequence $z_{n} \rightarrow 0$ which satisfies the conditions of the lemma.

Lemma 4. Suppose that $f$ is not an exceptional function from Lemma 2. Let $p, Q$ and $\mathcal{T}: \mathcal{O}_{0} \rightarrow \mathcal{O}_{1}$ be as above and let $L$ be the line through 0 and $Q$. Let $\gamma$ be the $\operatorname{arc} \Psi\left(L \cap \mathcal{O}_{0}\right)$. Then:

1. $\gamma$ is an unstable manifold for the fixed point $p$;

2. there exists a sequence $z_{n} \in L, z_{n} \rightarrow 0$, so that $\Psi\left(z_{n}\right)$ is a periodic point of period $n$ and $\gamma$ is an unstable manifold for $\Psi\left(z_{n}\right)$; 
3. for each $n$ large enough, $\gamma$ is an invariant curve for $\Psi\left(\lambda^{-n} Q\right)$ (which is in the backward orbit of $p)$;

4. $\Psi\left(\mathcal{O}_{0} \cap L\right)=\Psi\left(\mathcal{O}_{1} \cap L\right)$, and so $\mathcal{T}$ maps $\mathcal{O}_{0} \cap L$ diffeomorphically onto $\mathcal{O}_{1} \cap L$;

5. the set $\left\{z \in \mathcal{O}_{0}: D \mathcal{T}(z) \in \mathbb{R}\right\}$ is a finite union of real analytic curves, one of which is $\mathcal{O}_{0} \cap L$.

Proof. First we prove that

$$
D \mathcal{T}\left(z_{n}\right) \in \mathbb{R}
$$

for the points $z_{n}$ from Lemma 3. Let $x_{n}=\Psi\left(z_{n}\right)$ be the corresponding periodic points of period $n$. Then $\Psi \lambda^{n}=f^{n} \Psi$ implies

$$
D \Psi\left(\lambda^{n} z_{n}\right) \lambda^{n}=D f^{n}\left(x_{n}\right) D \Psi\left(z_{n}\right) .
$$

Since $D f^{n}\left(x_{n}\right)$ and $\lambda$ are real, it follows that $D \Psi\left(\lambda^{n} z_{n}\right) / D \Psi\left(z_{n}\right) \in \mathbb{R}$. We note that $\Psi\left(\lambda^{n} z_{n}\right)=f^{n}\left(\Psi\left(z_{n}\right)\right)=f^{n}\left(x_{n}\right)=x_{n}=\Psi\left(z_{n}\right)$ and $z_{n} \rightarrow 0$ and $\lambda^{n} z_{n} \rightarrow Q$, and therefore

$$
\mathcal{T} z_{n}=\lambda^{n} z_{n}
$$

Hence $D \Psi\left(\mathcal{T} z_{n}\right) / D \Psi\left(z_{n}\right) \in \mathbb{R}$. This implies (1).

If $D \mathcal{T}$ is constant on $\mathcal{O}_{0}$, then $\mathcal{T}$ is an affine map, $\mathcal{T}(z)=a z+Q$, where $a=D \mathcal{T}(0) \in \mathbb{R} \backslash\{0\}$. The identity $\Psi \circ \mathcal{T}=\Psi$ with a non-constant meromorphic function $\Psi$ implies that $a= \pm 1$, and we conclude that $\Psi$ is periodic. Then $f$ is an exceptional function from Lemma 2, contrary to our assumption.

From now on we assume that $D \mathcal{T}$ is not constant. Then the set $X=\left\{z \in \mathcal{O}_{0}\right.$ : $D \mathcal{T} \in \mathbb{R}\}$ is a finite union of real analytic curves. We are going to prove that $L \cap \mathcal{O}_{0}$ is one of these curves.

Without loss of generality we may assume that $L$ is the real line.

Let $\beta$ be a curve in $X$ that contains infinitely many points $z_{n}$. As $\lambda^{n} z_{n} \rightarrow Q$, we conclude $\arg z_{n} \rightarrow 0$, so $\beta$ is tangent to $L$ at 0 . If $\mathcal{T}$ is real or if $\beta \subset L$, then we are done.

So suppose that $\mathcal{T}$ is not real, and write

$$
\mathcal{T}(z)=Q+a_{1} z+\ldots+a_{m} z^{m}+a_{m+1} z^{m+1}+O\left(z^{m+2}\right), \quad z \rightarrow 0,
$$

where $m$ is chosen so that $a_{1}, \ldots, a_{m}$ are real, while $a_{m+1}$ is not real. Since $D \mathcal{T}(0) \in$ $\mathbb{R} \backslash\{0\}$, we have $m \geq 1$. Our curve $\beta$ is tangent to the real line $L$ at 0 and is contained in the set $\{z: D \mathcal{T} \in \mathbb{R}\}$. This curve $\beta$ has the form $\beta(x)=x+i b x^{K}+o\left(x^{K}\right)$, and we are assuming that $b \neq 0$ and $K>1$. Let $k \geq 2$ be the smallest subscript for which $a_{k} \neq 0$. The condition $\left.D \mathcal{T}\right|_{\beta} \in \mathbb{R}$ gives, when $k<m+1$,

$$
\Im D \mathcal{T}(\beta(x))=k(k-1) a_{k} b x^{k-2+K}+(m+1) \Im a_{m+1} x^{m}+\ldots \equiv 0,
$$

where we use that $\left(x+i b x^{K}+o\left(x^{K}\right)\right)^{k-1}=x^{k-1}+i(k-1) x^{k-2} b x^{K}+o\left(x^{k-2+K}\right)$. If $k \geq m+1$, then $\left.D \mathcal{T}\right|_{\beta} \in \mathbb{R}$ gives $\Im D \mathcal{T}(\beta(x))=(m+1) \Im a_{m+1} x^{m}+\ldots \equiv 0$, which is impossible. From (3) it follows that $k-2+K=m$; here we used that $a_{m+1}$ is not real. So

$$
m \geq K \text {. }
$$

Since $z_{n} \in \beta$ is of the form $t_{n}+i b t_{n}^{K}+o\left(t_{n}^{K}\right)$, we have $\Re \mathcal{T} z_{n} \rightarrow Q \neq 0$, and $\Im \mathcal{T} z_{n}=O\left(t_{n}^{K}\right)$ in view of (4). So $\arg z_{n} \sim t_{n}^{K-1}$ while $\arg \mathcal{T}\left(z_{n}\right)=O\left(t_{n}^{K}\right)$, which contradicts (2).

This proves property 5 of the lemma. 
Now $\mathcal{T}: \mathcal{O}_{0} \rightarrow \mathcal{O}_{1}$ is biholomorphic, $\mathcal{T}(0)=Q$ and $D \mathcal{T}(z)$ is real for real $z$. This implies property 4.

We put $\gamma=\Psi\left(L \cap \mathcal{O}_{0}\right)$. Then property 1 is evident: take $\gamma_{*}=\Psi\left(\lambda^{-1}\left(L \cap \mathcal{O}_{0}\right)\right)$. Property 2 follows from $\lambda^{n}\left(V_{n} \cap L\right)=\mathcal{O}_{1} \cap L$ (notation from Lemma 3 ) and the fact that $f^{n}: \Psi\left(V_{n}\right) \rightarrow \Psi\left(\mathcal{O}_{0}\right)$ is biholomorphic. This also implies property 3 because $\lambda^{-n} Q \in V_{n}$.

1.2. The case that $\Psi^{-1}(J(f))$ is not contained in a line. We will use the following notation: if $\gamma$ is a curve through $x$, then $T_{x} \gamma$ will denote its tangent line at $x$.

Lemma 5. Assume that $\Psi^{-1}(J(f)) \not \subset L$. Then $\Psi$ is a periodic function and $f$ is a Lattès map.

Proof. Throughout the proof, $Q, \Psi$ and $\mathcal{T}: \mathcal{O}_{0} \rightarrow \mathcal{O}_{1}$ will be as previously defined in Lemma 3 (with $\mathcal{O}_{1}$ a neighborhood of $Q$ ).

Since $\Psi^{-1}(J(f)) \not \subset L$, there exists $Q^{1} \in \mathbb{C} \backslash L$ so that $\Psi\left(Q^{1}\right)$ is in the backward orbit of $p$, say $f^{m}\left(\Psi\left(Q^{1}\right)\right)=p$. Define $Q^{\prime}=\lambda^{m} Q^{1}$; then

$$
\Psi\left(Q^{\prime}\right)=\Psi\left(\lambda^{m} Q^{1}\right)=f^{m} \Psi\left(Q^{1}\right)=p
$$

and thus Lemma 4 applies to the line $L^{\prime}$ through 0 and $Q^{\prime}$.

As we assume that $\Psi^{-1}(J(f)) \notin L$, there is an infinite set of lines $L^{\prime}$ as above. Indeed, it is easy to see that whenever $\Psi^{-1}(J(f))$ is contained in a finite union of lines, it is actually contained in one line. We are going to prove that $D \mathcal{T}(z) \in$ $\mathbb{R}, \forall z \in L^{\prime}$, for each such line $L^{\prime}$, thus concluding from part 5 of Lemma 4 that $f$ is one of the functions listed in Lemma 2. For all those functions, except Lattès maps, $\Psi(J(f))$ is a line, so we will conclude that $f$ is a Lattès map.

We denote by $\mathcal{O}_{0}^{\prime}$ and $\mathcal{O}_{1}^{\prime}$ neighborhoods of 0 and $Q^{\prime}$, respectively, such that $\Psi$ is univalent in these neighborhoods and $\Psi\left(\mathcal{O}_{0}^{\prime}\right)=\Psi\left(O_{1}^{\prime}\right)$. We choose a round disc as $\mathcal{O}_{0}^{\prime}$.

Applying Lemmas 3 and 4 to the point $Q^{\prime}$ we obtain a sequence $z_{k} \in L^{\prime}, z_{k} \rightarrow 0$ such that $\lambda^{k} z_{k} \rightarrow Q^{\prime}$ and $x_{k}=\Psi\left(z_{k}\right)$ are repelling periodic points of period $k$. Fix such a point $z=z_{k} \in \mathcal{O}_{0}^{\prime}$ so that $\lambda^{k} z \in \mathcal{O}_{1}^{\prime}$, and $x=\Psi(z)$ is the corresponding periodic point (of period $k$ ) which does not belong to the forward orbit of a critical point.

By statements 1, 2 and 4 of Lemma $4, \gamma^{\prime}:=\Psi\left(L^{\prime} \cap \mathcal{O}_{0}^{\prime}\right)=\Psi\left(L^{\prime} \cap \mathcal{O}_{1}^{\prime}\right)$ is an unstable manifold for $p$ and also an unstable manifold for $x$. Since $\Psi \mid \mathcal{O}_{0}^{\prime}$ is univalent, the curve $\gamma^{\prime}$ is smooth and has no self-intersections. (We chose $\mathcal{O}_{0}^{\prime}$ to be a disc, and so $\gamma^{\prime}$ is connected.) Since $\gamma^{\prime}$ is an unstable manifold of $x$, there exists a curve $\gamma_{*}^{\prime} \subset \gamma^{\prime}$ through $x$ so that $f^{k}$ maps $\gamma_{*}^{\prime}$ diffeomorphically onto $\gamma^{\prime}$. That is, there exists a nested sequence of curves $\gamma_{i, *}^{\prime} \supset \gamma_{i+1, *}^{\prime} \ni x$ shrinking in diameter to 0 (with $\left.\gamma_{0, *}^{\prime}=\gamma^{\prime}\right)$ so that $f^{k}$ maps $\gamma_{i+1, *}^{\prime}$ diffeomorphically onto $\gamma_{i, *}^{\prime}$.

Now also consider the linearization $\hat{\Psi}$ of $f^{k}$ associated to the periodic point $x$, i.e.

$$
f^{k} \hat{\Psi}=\hat{\Psi} \mu \quad \text { where } \quad \mu=D f^{k}(x) .
$$

Let $\gamma_{i, *}^{\prime}$ be the arcs defined a few lines above, and take $i$ so large that there exists a curve $\hat{L}_{i}^{\prime}$ containing 0 which is mapped by $\hat{\Psi}$ diffeomorphically onto $\gamma_{i, *}^{\prime}$. Note that $f^{i k}: \gamma_{i, *}^{\prime} \rightarrow \gamma^{\prime}$ can be written as $\hat{\Psi} \circ \mu^{i} \circ\left(\hat{\Psi} \mid \hat{L}_{i}^{\prime}\right)^{-1}$, and, since this map is a diffeomorphism onto, it follows that $\hat{\Psi}$ is also a diffeomorphism restricted to the 
curve $\hat{L}^{\prime}:=\mu^{i} \hat{L}_{i}^{\prime}$ and that $\hat{\Psi}\left(\hat{L}^{\prime}\right)=\gamma^{\prime}$. In particular, there exists $\hat{w} \in \hat{L}^{\prime}$ so that $\hat{\Psi}(\hat{w})=p$.

Since $\gamma^{\prime}$ is an unstable manifold for $x$, the curve $\hat{L}^{\prime}$ is invariant under $z \mapsto \mu z$. As the only smooth curve through 0 which is invariant under real multiplication is a line, $\hat{L}^{\prime}$ must be contained in a line $\hat{M}$ through 0 .

Let $z^{\prime}=\mathcal{T}(z) \in \mathcal{O}_{1}$. For $j \geq 0$ large, $w_{j}:=\lambda^{-j k}\left(z^{\prime}\right)$ is contained in $\mathcal{O}_{0}$. Note that $\Psi\left(w_{j}\right)$ tends to $p=\hat{\Psi}(\hat{w})$ as $j \rightarrow \infty$. Since $\hat{\Psi}$ is a diffeomorphism restricted to $\hat{L}^{\prime}$, and $\hat{w} \in \hat{L}^{\prime}$, then for $j$ large enough there exist unique $\hat{w}_{j}$ near $\hat{w}$ such that $\hat{\Psi}\left(\hat{w}_{j}\right)=\Psi\left(w_{j}\right)$.

Note that

$$
\hat{\Psi}\left(\mu^{j} \hat{w}_{j}\right)=f^{j k} \hat{\Psi}\left(\hat{w}_{j}\right)=f^{j k} \Psi\left(w_{j}\right)=\Psi\left(\lambda^{j k} w_{j}\right)=\Psi\left(z^{\prime}\right)=\Psi(z)=x .
$$

Let $\hat{M}_{j}^{\prime}$ be the line through 0 and $\mu^{j} \hat{w}_{j}$ and let $\hat{M}_{j} \subset \hat{M}_{j}^{\prime}$ be an open line segment containing the line segment $\left[\hat{w}_{j}, 0\right]$ and contained in a small neighborhood of $\left[\hat{w}_{j}, 0\right]$. By Lemma 1, there exist neighborhoods $\hat{\mathcal{O}}_{0} \ni 0$ and $\hat{\mathcal{O}}_{1} \ni \mu^{j} \hat{w}_{j}$ on each of which $\hat{\Psi}$ is biholomorphic, and $\hat{\Psi}\left(\hat{\mathcal{O}}_{0}\right)=\hat{\Psi}\left(\hat{\mathcal{O}}_{1}\right)$. Next apply Lemma 4 to the map $\hat{\Psi}$ (taking instead of $L, Q$ the line $\hat{M}_{j}^{\prime}$ and $\left.\mu^{j} \hat{w}_{j} \in \hat{\Psi}^{-1}(x)\right)$. This gives that $\hat{\Psi}\left(\hat{M}_{j}^{\prime} \cap \hat{\mathcal{O}}_{0}\right)$ is an invariant manifold for $x$ and that $\hat{\Psi}\left(\hat{M}_{j}^{\prime} \cap \hat{\mathcal{O}}_{0}\right)=\hat{\Psi}\left(\hat{M}_{j}^{\prime} \cap \hat{\mathcal{O}}_{1}\right)$. By statements 3 and 4 of Lemma 4 , there exist small neighborhoods $\hat{V}_{j}$ of $\hat{w}_{j}, \hat{V}_{j}^{1}$ of $\mu^{j} \hat{w}_{j}$ and $\hat{V}_{j}^{0}$ of 0 so that

$$
f^{j k}\left(\hat{\Psi}\left(\hat{M}_{j}^{\prime} \cap \hat{V}_{j}\right)\right)=\hat{\Psi}\left(\hat{M}_{j}^{\prime} \cap \hat{V}_{j}^{1}\right)=\hat{\Psi}\left(\hat{M}_{j}^{\prime} \cap \hat{V}_{j}^{0}\right) \subset \hat{\Psi}\left(\hat{M}_{j}\right) .
$$

The first equality holds in view of (5) since $\mu$ is real. Since $\hat{w}_{j}$ lies close to $\hat{w}$ and $\hat{\Psi}$ is a diffeomorphism restricted to $[\hat{w}, 0], \quad \hat{\Psi}\left(\hat{M}_{j}\right)$ is a smooth curve which lies close to $\hat{\Psi}\left([\hat{w}, 0]\right.$ ) (which is the subarc of $\gamma^{\prime}$ connecting $p$ and $x$ defined by $\Psi([0, z]))$. It follows that there exists a curve $M_{j} \subset \mathcal{O}_{0}$ through $w_{j}$ and $z$ so that $\Psi\left(M_{j}\right)=\hat{\Psi}\left(\hat{M}_{j}\right)$. By (6) , there exists a small neighborhood $V_{j}$ of $w_{j}$ so that

$$
\Psi\left(\lambda^{j k}\left(M_{j} \cap V_{j}\right)\right)=f^{j k}\left(\Psi\left(M_{j} \cap V_{j}\right)\right) \subset \Psi\left(M_{j}\right)=\Psi\left(\mathcal{T} M_{j}\right) .
$$

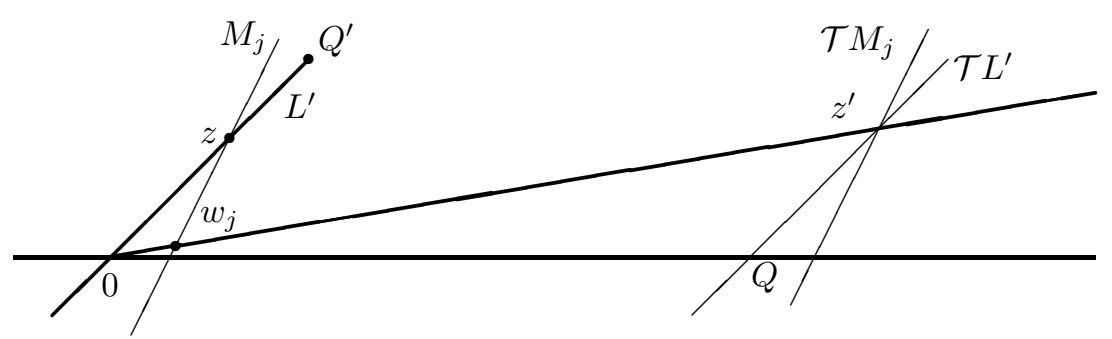

Figure 1. The curves used in the proof of Lemma 5, The thinly drawn curves are not necessarily line segments.

Since $\lambda^{j k} w_{j}=z^{\prime}$, the curves $\lambda^{j k} M_{j}$ and $\mathcal{T} M_{j}$ both go through $z^{\prime}$, and by the previous inclusion these curves agree near $z^{\prime}$. In particular, the tangents of these 
curves at $z^{\prime}$ agree:

$$
T_{z^{\prime}}\left(\lambda^{j k} M_{j}\right)=T_{z^{\prime}}\left(\mathcal{T} M_{j}\right) .
$$

The left hand side of (7) is equal to $T_{w_{j}} M_{j}$. Note that $\hat{M}_{j}$ converges to $\hat{M}$ as $j \rightarrow \infty$, that $\hat{\Psi}\left(\hat{L}^{\prime}\right)=\gamma^{\prime}=\Psi\left(L^{\prime} \cap \mathcal{O}_{0}^{\prime}\right)$ with $\hat{L}^{\prime} \subset \hat{M}$, and that $\Psi\left(M_{j}\right)=\hat{\Psi}\left(\hat{M}_{j}\right)$. Hence $M_{j}$ converges in the $C^{1}$ sense to a segment in $L^{\prime}$. (Here we use that $\hat{\Psi}$ is a diffeomorphism on a neighborhood of $[0, \hat{w}]$.

We therefore get that $T_{z^{\prime}}\left(\lambda^{j k} M_{j}\right) \rightarrow T_{0} L^{\prime}=T_{z} L^{\prime}$ and that the right hand side of (7) converges to $T_{z^{\prime}}\left(\mathcal{T} L^{\prime}\right)$. Combined, it follows that

$$
T_{z}\left(L^{\prime}\right)=T_{z^{\prime}}\left(\mathcal{T} L^{\prime}\right)
$$

and so $D \mathcal{T}(z) \in \mathbb{R}$. Since this holds for a whole sequence of points $z=z_{k} \in L^{\prime}$ we obtain that $D \mathcal{T}(z) \in \mathbb{R}$ for all $z \in L^{\prime}$.

As there are infinitely many such lines $L^{\prime}$, this implies that $D \mathcal{T}$ is constant; thus $f$ is a Lattès map.

1.3. Completion of the proof of Theorem 1. If $f$ is not a Lattès map, Lemma 5 implies that $\Psi^{-1}(J(f)) \subset L$. Without loss of generality we may assume that $L$ is the real line.

We recall that the order $\rho$ of a meromorphic function $\Psi$ is defined by the formula

$$
\rho=\limsup _{r \rightarrow \infty} \frac{\log T(r, f)}{\log r},
$$

where $T(t, f)$ is the Nevanlinna characteristic 9 . According to a theorem of Valiron [11, §51], the order of a Poincaré function $\Psi$ satisfying the equation

$$
\Psi \lambda=f^{N} \Psi
$$

can be found by the formula

$$
\rho=N \log \operatorname{deg} f / \log |\lambda| .
$$

We claim that under the assumption that $J \subset \Psi(L)$, one can always find infinitely many periodic points $p$ such that the orders of the corresponding functions $\Psi$ will satisfy $\rho \leq 1+\epsilon$, for any given $\epsilon>0$.

To prove the claim, we consider the measure of maximal entropy $\mu$ and the characteristic exponent

$$
\chi(z)=\lim _{n \rightarrow \infty} \frac{1}{n} \log \left|\left(D f^{n}\right)(z)\right| .
$$

The reader may consult the survey [2] about these notions. According to the multiplicative ergodic theorem, this limit exists a.e. with respect to $\mu$, and it is equal a.e. to the average characteristic exponent

$$
\chi:=\int \log |D f(z)| d \mu(z) .
$$

The average characteristic exponent is related to the Hausdorff dimension $H D(\mu)$ of measure $\mu$ by the formula

$$
\chi=\frac{\log \operatorname{deg} f}{H D(\mu)},
$$

proved in [7]. As $\mu$ is supported on the Julia set, and the Julia set is the image of a line under a meromorphic function, we conclude that $H D(\mu) \leq 1$. So

$$
\chi \geq \log \operatorname{deg} f .
$$


Now, $\mu$ is a weak limit of atomic probability measures $\mu_{N}$ equidistributed over periodic points of period $N$. Then (9) and (10) imply that there are infinitely many periodic points $p$ of periods $N$ such that the multipliers $\lambda$ of these points satisfy $\log |\lambda| \geq(1-\epsilon) N(\log \operatorname{deg} f)$. We conclude from Valiron's formula (8) that the order of the Poincaré function $\Psi$ is at most $(1-\epsilon)^{-1}$, as advertised.

We may assume without loss of generality that $\{0, \infty\} \subset J(f)$ and that $p=$ $\Psi(0) \in \mathbb{R}$. (This can be achieved by conjugating $f$ by a fractional-linear transformation.) As we also assume that $L=\mathbb{R}$, the zeros $a_{j}$ and poles $b_{j}$ of $\Psi$ are all real. Taking $\epsilon=1 / 3$ we obtain a Poincaré function of order at most 3/2. According to a theorem of Nevanlinna [5, 6, 9, our function $\Psi$ of order less than 2 has a canonical representation

$$
\Psi(z)=b e^{a z} \frac{\prod_{j}\left(1-z / a_{j}\right) e^{z / a_{j}}}{\prod_{j}\left(1-z / b_{j}\right) e^{z / b_{j}}} .
$$

As $b=\Psi(0)$, and $a_{j}$ and $b_{j}$ are all real, we conclude

$$
\Psi(z)=e^{i c z} g(z),
$$

where the function $g$ is real on the real line and the constant $c=\Im a$ is real. If $c=0$, then $\Psi(\mathbb{R})$ is contained in the real line, and this completes the proof.

Suppose now that $c \neq 0$. We assume as before that the point $p$ does not belong to the critical orbit of $f$. Then $p$ is not a critical value of $\Psi$. Suppose that $\Psi\left(z_{n}\right)=p$ for $n=1,2, \ldots$; then all $z_{k}$ are real. Put $\Psi_{n}(z)=\Psi\left(z+z_{n}\right)$. Let $U_{n}$ be small intervals around zero on the real line such that the $\Psi_{n}$ are univalent in $U_{n}$. Let $\gamma_{n}=\Psi_{n}\left(U_{n}\right)$. These are analytic curves, and (since the Julia set is perfect) any two of them have infinitely many intersection points having an accumulation at $p=\Psi(0)$. We conclude that all these $\gamma_{n}$ are reparametrizations of the same curve: $\gamma_{n}=\gamma$. Now each function $\Psi_{n}$ maps $U_{n}$ to the same curve $\gamma$, and (11) implies that the rate of change of the arguments of $\Psi_{n}(x)$ is the same non-zero constant $c$. We conclude that all $\Psi_{n}$ are equal, which implies that $\Psi$ is a periodic function. According to Lemma 2 this can happen only if $f$ is conjugated to $z^{d}$ or to a Chebyshev polynomial or to a Lattès map. This proves our theorem in the case that $c \neq 0$ and thus completes the proof.

\section{Rational Functions with Real Julia Sets}

Proof of Theorem 2. Evidently $f(C) \subset C$. If there are no critical points on $C$, then the restriction $f: C \rightarrow C$ is a covering. The degree of this covering must be equal to $\operatorname{deg} f$ since every point of the Julia set has $\operatorname{deg} f$ preimages in $C$. Thus $C$ is completely invariant and $f^{2}$ is a Blaschke product. From now on we assume that $f$ has a critical point on $C$.

If $J(f)=C$, then both components of the complement of $C$ are invariant under $f^{2}$, so $f^{2}$ is a Blaschke product in this case as well.

If $J(f) \neq C$, the set of normality is connected, thus there is a fixed point $z_{0}$ to which the iterates on the set of normality converge. As $f(C) \subset C, f$ commutes with reflection with respect to $C$. This implies that $z_{0} \in C$. Evidently, the multiplier of $z_{0}$ satisfies $-1 \leq \lambda \leq 1$.

We may assume without loss of generality that $C=\overline{\mathbb{R}}$ and $z_{0}=\infty$. Let $I=[a, b]$ be the convex hull of the Julia set. This means that $\overline{\mathbb{R}} \backslash I$ is the immediate basin of 
attraction of $\infty$ for the restriction $\left.f\right|_{\overline{\mathbb{R}}}$. As the boundary of the immediate basin is invariant, we obtain

Lemma 6. The set $\{a, b\}$ is $f$-invariant. If $f([a, b]) \subset[a, b]$, then $J(f)=[a, b]$.

If $f([a, b]) \not \subset[a, b]$, then there exists an interval $(\alpha, \beta) \subset[a, b]$ which is mapped by $f$ outside $[a, b]$ (and $\alpha, \beta$ are mapped into $a$ or $b$ ). Since the preimages of $\alpha, \beta$ are dense in the Julia set, it follows that in this case the Julia set is a Cantor set.

Lemma 7. Each critical point of $f$ in I is contained in the closure of a real interval which is a component of the basin of $z_{0}$. In particular, each critical point in the Julia set is preperiodic.

Proof. Let us call a point $x_{0} \in J(f)$ an endpoint of $J(f)$ if $J(f)$ accumulates to $x_{0}$ only from one side (left or right). It is clear that the endpoints of $J(f)$ are boundary points of the immediate basin of $z_{0}$ on $\overline{\mathbb{R}}$. On the other hand, it is easy to see that all critical points must be of odd degree, and if $x$ is a critical point and $c=f(x)$ is the corresponding critical value, then one of the equations $f(x)=c+\epsilon$ and $f(z)=c-\epsilon$ has non-real solutions in a neighborhood of $x$ for all sufficiently small $\epsilon$. Thus the critical value $c \in J$ has to be an endpoint of $J(f)$.

So for each critical point $x \in(a, b)$ of $f$ there exists an integer $N \geq 1$ with $f^{N}(x) \notin(a, b)$.

This proves Theorem 2 .

2.1. Polynomials with real Julia sets. For polynomials with real Julia sets, a complete parametric description is possible.

Let $f$ be a polynomial of degree $d$ whose Julia set $J$ is real. We may assume that the convex hull of $J$ is $[0,1]$. Then all $d$ zeros of $f$ are real (belong to $[0,1]$ ). Thus all critical points are also real and belong to $[0,1]$. Let $c_{1}, \ldots, c_{d-1}$ be the critical values enumerated left to right. Then the condition that the equation $f(z)=1$ has all solutions real implies that all $c_{j}$ are outside the interval $(0,1)$. Moreover, we obtain for odd $d$ that 0 and 1 are either fixed or make a 2-cycle. For even $d$ we have $f(0)=f(1) \in\{0,1\}$.

Now, critical values of such polynomials satisfy

$$
(-1)^{j} c_{j} \quad \text { is of constant sign. }
$$

This solves the classification problem completely. We can prescribe arbitrarily $d-1$ critical values $c_{j} \in \mathbb{R} \backslash(0,1)$ satisfying (12). Then there exists a real polynomial with these critical values (ordered sequence!). This polynomial is unique up to the change of the independent variable $z \mapsto a z+b$ with positive $a$ and real $b$. Using this change of the variable we achieve that the convex hull of the set $\{z: f(z) \in\{0,1\}\}$ is $[0,1]$.

Thus there is a bijective correspondence between sequences of critical values $\left(c_{1}, \ldots, c_{d-1}\right)$ satisfying $c_{j} \in \mathbb{R} \backslash(0,1)$ and (12) and polynomials with the property that the convex hull of the Julia set is $[0,1]$. Chebyshev polynomials correspond to the case $c_{j} \in\{0,1\}$. All other polynomials of our class have Cantor Julia sets.

2.2. Rational functions of the class (iii) in Theorem $\mathbf{2}$. We were unable to give any classification of these functions, so we only give several examples.

Example 1. The simplest non-polynomial example of case (iii) is a perturbation of a quadratic polynomial. Consider $f(z)=\left(z^{2}-4\right) /(1+c z)$ with $c \in \mathbb{R}$. If $|c|<1$, 
this map has an attractor at $\infty$ with multiplier $c$. Note that $f^{\prime}(z)=\frac{c z^{2}+2 z+4 c}{(1+c z)^{2}}$ and that this has two real zeros when $-1 / 2<c<1 / 2$. To compute $f^{-1}(\mathbb{R})$, we note that $f(z)=w$ is equivalent to

$$
z=\frac{c w \pm \sqrt{c^{2} w^{2}+4 w+16}}{2} .
$$

It follows that when $|c|>1 / 2, c \in \mathbb{R}$, then $f^{-1}(\mathbb{R}) \subset \mathbb{R}$ and so $f$ is a Blaschke product, while for $|c|<1 / 2, c \in \mathbb{R}$, we find $f^{-1}(\mathbb{R}) \not \subset \mathbb{R}$ and so $f$ is not a Blaschke product. Note that $f$ is a Blaschke product with an attracting fixed point at $\infty$ if $c \in \mathbb{R}$ and $1 / 2<|c|<1$.

As remarked, for $|c|<1 / 2, f$ is not a Blaschke product. Let us determine its Julia set. There exists an interval $I=[p, q]$ containing 0 so that $f(p)=f(q)=q$, $f: I \rightarrow \mathbb{R}$ is continuous and has a minimum at some $c \in \operatorname{int}(I)$ with $f(c)<p$. Hence there exist two disjoint intervals $I_{0}, I_{1}$ in $I$ which are mapped diffeomorphically onto $I$, and so $f$ has a full horseshoe $\Lambda$ in $[p, q]$. Each other point is in the basin of the attractor at $\infty$. Since $f$ has degree two, this horseshoe is also backward invariant, $f^{-1}(\Lambda)=\Lambda$ and so it follows that $J(f)=\Lambda \subset \mathbb{R}$.

Example 2. A function of the type (iii) can have a neutral rational fixed point. Indeed, take $f(z)=\frac{(z-2)(z+c)(z-c)}{(z-1)(z+1)}$ with $c \in(0,1)$ close to 1 . Then $\infty$ is a parabolic fixed point which attracts real points $x \in(-\infty,-1)$ and repels points with $x \in \mathbb{R}$ and $x$ large. (Indeed, $f(x)<x$ for $x \in \mathbb{R}$ and $|x|$ large because $\frac{(x+c)(x-c)}{(x-1)(x+1)}>1$ for $|x|>1$, and therefore $f(x)<x$ when $x \in(-\infty,-1)$. A similar argument shows that $f(x)<x$ when $x \in(1, \infty)$.) The map $f$ has a unique minimum $c \in(-1,1)$ with $f(c)<-1$. There are three disjoint intervals $I_{1}, I_{2}, I_{3}$ with $I_{1}, I_{2} \subset(-1,1)$ and $I_{3} \subset(1, \infty)$ such that $f$ maps each of these diffeomorphically onto $(-1, \infty)$. So the Julia set contains a set $\Lambda \subset(-1, \infty)$ on which $f$ acts as a subshift of three symbols. Since $f$ has degree 3 , it follows that each preimage of this interval again lies inside this interval. Hence $J(f)=\Lambda \subset \mathbb{R}$. Each point outside $\Lambda$ is in the basin of $\infty$. Clearly $f$ is not a Blaschke product (there exist critical points on $\mathbb{R}$ so $f^{-1}(\mathbb{R})$ is not contained in $\mathbb{R}$ ).

Our last example shows that in general one cannot take $N=1$ in case (iii) of Theorem 2.

Example 3. We begin with a Blaschke product of degree 2,

$$
g(z)=K z \frac{z-a}{z-p}, \quad 0<p<a<1
$$

where the constants are chosen such that $K>1, f(1)=1$, and $f^{\prime}(1)>1$. This function has two branches defined on subintervals of $[0,1]$ that map each subinterval on the whole $[0,1]$, so the Julia set is a Cantor set whose convex hull is [0,1]. Fix a closed interval $I \subset(p, a)$ on which $f(x) \leq-1$ (i.e. $I$ is in the basin of the attractor at infinity), and let $c$ be the middle point of this interval. Let $b$ be the preimage of the point $c$ on the interval $[a, 1]$. Now we make a small perturbation of $g$ so that the resulting rational function of degree 3 is very close to $g$ on $[0,1]$ minus a small 
neighborhood of the point $b$. Our function is

$$
f(z)=K(\epsilon) g(z) \frac{z-b+\epsilon}{z-b-\epsilon},
$$

where $\epsilon$ is a very small positive number and $K(\epsilon)$ is chosen so that $f(1)=1$, so that $K(\epsilon) \rightarrow 1$ as $\epsilon \rightarrow 0$. It is clear that $f$ has two critical values $c_{1}<c_{2}$ on $[0,1]$ at the critical points near $b$.

It is also easy to see that these critical values both tend to $c$ as $\epsilon \rightarrow 0$. (Indeed, fix $\delta>0$ small and let $V=[b-\delta, b+\delta]$ be a small neighborhood of $b$. Our function $f$ converges to $g$, and also $f^{\prime}$ converges to $g^{\prime}$ outside $V$ (as $\epsilon \rightarrow 0$ ). In particular, $f(b+\delta)$ is close to $c=g(b)$ and $f$ is increasing at this point $b+\delta$. But $f$ also has a pole at $b+\epsilon<b+\delta$, and it is decreasing on the right hand side of this pole. It follows that $f$ has a critical point (a minimum) on the interval $[b+\epsilon, b+\delta]$ with critical value at most $g(b+\delta)+\delta$ (when $\epsilon>0$ is close to zero), which is close to $c=g(b)$. There is also another critical point on the other side of $b$, where the critical value is greater than $g(b-\delta)-\delta$. As the right critical value is evidently greater than the left one, both critical values tend to $c=g(b)$.)

So if $\epsilon$ is small enough, we have $f\left(\left[c_{1}, c_{2}\right]\right) \cap I=$, thus the whole interval $\left[c_{1}, c_{2}\right]$ escapes from $[0,1]$ under the second iterate of $f$. We also conclude that $J(f) \subset[0,1]$, because each point of $[0,1] \backslash\left[c_{1}, c_{2}\right]$ has three preimages in $[0,1] \backslash\left[c_{1}, c_{2}\right]$.

\section{REFERENCES}

[1] W. Bergweiler and A. Eremenko, Meromorphic functions with linearly distributed values and Julia sets of rational functions, Proc. AMS, 137 (2009) 2329-2333. MR2495266 (2010d:30036)

[2] A. Eremenko and M. Lyubich, Dynamics of analytic transformations, Leningrad Math. J., 1 (1990) 563-634. MR1015124(91b:58109)

[3] P. Fatou, Sur les équations fonctionnelles. Premiere mémoire, Bull. Soc. Math. France, 47 (1919) 161-271. MR1504787

[4] P. Fatou, Sur les équations fonctionnelles. Troisième mémoire, Bull. Soc. Math. France, 48 (1920) 208-314. MR 1504797

[5] A. Goldberg and I. Ostrovskii, Value distribution of meromorphic functions, AMS, Providence, RI, 2008. Troisieme memoire, Bull. Soc. Math. France, 48 (1920) 208-314. MR 2435270 (2009f:30067)

[6] W. Hayman, Meromorphic functions, Clarendon Press, Oxford, 1964. MR0164038 (29:1337)

[7] F. Leddrapier, Quelques propriétés ergodiques des applications rationnelles, C. R. Acad. Sci., 299 (1984) 37-40. MR756305 (86c:58091)

[8] J. Milnor, Dynamics in One Variable, Princeton Univ. Press, Princeton, NJ, 2006. MR2193309 (2006g:37070)

[9] R. Nevanlinna, Analytic functions, Springer, NY, 1970. MR0279280 (43:5003)

[10] J. F. Ritt, Periodic functions with a multiplication theorem, Trans. Amer. Math. Soc., 23 (1922) 16-25. MR.1501186

[11] G. Valiron, Fonctions analytiques, Presses universitaires de France, Paris, 1954. MR 0061658 $(15: 861 \mathrm{a})$

Department of Mathematics, Purdue University, West Lafayette, Indiana 47907

E-mail address: eremenko@math.purdue.edu

Department of Mathematics, University of Warwick, Coventry CV4 7AL, United KINGDOM

E-mail address: strien@maths.warwick.ac.uk 